\title{
On the Set Representation of Fuzzy Random Vectors Obtained as Vague Perception of Random Phenomena
}

\author{
Tokuo Fukuda \\ Faculty of Management, Otemon Gakuin University, \\ 2-1-15 Nishi-Ai, Ibaraki, Osaka, 567, JAPAN
}

\begin{abstract}
In this paper, the author tries to reconstruct fuzzy random vectors(FRVCs) by using set representation approach. Fuzzy random vectors as vague perception of random phenomena is proposed by author, which have intrinsically both properties of fuzziness and randomness and they are obtained as fuzzy perceptions of ordinary non-fuzzy random vectors. First, FRVCs are re-defined by using the set representation of fuzzy sets. Secondly, adopting the multi-valued logic, the expectation of FRVCs are defined. Finally, some of the statistical properties of FRVCs are studied theoretically.
\end{abstract}

Keywords. Fuzzy random vectors, Set representation of fuzzy sets, Fuzzy set theory, Theory of correspondences

\section{Introduction}

Recently the author has proposed a class of fuzzy random vectors obtained as vague perception of random phenomena(Fukuda,1994,1995a,1995b), which have, of course, both properties of vagueness due to intrinsic ambiguous expression by using natural language and randomness due to the random fluctuation of object phenomena. In this paper, the author proposes to re-construct FRVCs by using set representation approach.

There have been already many literatures concerned with fuzzy random variables, which are closely related to FRVCs proposed in this paper. They are classified broadly into two types, i.e., one is those obtained by applying the multi-valued logic(Kwakernaak,1978; Miyakoshi and Shimbo,1984; Kruse and Meyer,1987; Boswell and Taylor,1987) and another is those obtained by extending the theory of correspondences or random sets(Klement,Puri and Ralescu,1986; Puri and Ralescu,1986; Inoue,1991; Umemura,1993). However, it may be fair to say that few effort have been made to investigate FRVCs from the consistent viewpoint of the fuzzy perceptions of non-fuzzy random vectors.

In this paper, using the set representation method of fuzzy sets, the FRVCs proposed by the author is re-constructed, and the expectation of the proposed FRVCs and some of their properties are investigated theoretically.

Section 2 devotes to describe the basic results concerned with general fuzzy sets. Re-constructed FRVCs are introduced in Sec. 3, where the methodology proposed by Kwakernaak $(1978)$ is basically adopted with the help of the theory of
correspondences(Klein and Thompson,1988). Furthermore, applying the concept of multi-valued logic, expectations of FRVCs are considered and some of their properties are also examined.

\section{Set Representations of Fuzzy Vectors}

In this paper, a fuzzy set $\tilde{U}$ given as a vague perception of $x_{0} \in \mathrm{X}$ is defined by the triple

$$
\tilde{U}=\left(\mathrm{X},\left\{\tilde{U}_{\alpha} \mid \alpha \in(0,1)\right\}, s_{\tilde{U}}\right),
$$

where $\mathrm{X}$ is called the basic space; $s_{\tilde{U}}$ is the proposition, i.e., $s: \mathrm{X} \rightarrow \mathcal{P}$ with $\mathcal{P}$ the "universe of discourse" defined by a set of statements, assigns a statement

$$
s_{\tilde{U}}(x)=\left\{x \text { coincides with } x_{\mathrm{o}}\right\}
$$

to each element $x \in \mathrm{X}$; and $\left\{\tilde{U}_{\alpha} \mid \alpha \in(0,1)\right\}$ is the family of subsets of $\mathrm{X}$ satisfying

$$
L_{\alpha} \tilde{U} \subseteq \tilde{U}_{\alpha} \subseteq L_{\bar{\alpha}} \tilde{U} \text { for } \forall \alpha \in(0,1) .
$$

In (3), $L_{\alpha} \tilde{U}$ and $L_{\bar{\alpha}} \tilde{U}$ are the strong cut and the level set of $\tilde{U}$ at the level $\alpha$ defined respectively by

$$
\begin{array}{ll}
L_{\alpha} \tilde{U}=\{x \mid(x \in \mathrm{X}) \wedge((\tilde{U})(x)>\alpha)\} & \text { for } \alpha \in[0,1) \\
L_{\bar{\alpha}} \tilde{U}=\{x \mid(x \in \mathrm{X}) \wedge((\tilde{U})(x) \geq \alpha)\} & \text { for } \alpha \in(0,1],
\end{array}
$$


where $(\tilde{U})(x)$ is the membership function of $\tilde{U}$ given by

$$
(\tilde{U})(x)=t\left(s_{\tilde{U}}(x)\right)
$$

and $t(*)$ in $(6)$ is the truth function of $*$ in the sense of multivalued logic. The crisp point $x_{\circ}$ in $\mathrm{X}$, which gives the vague perception, i.e., the fuzzy set $\tilde{U}$, is called the original point of $\tilde{U}$. The definition of fuzzy sets adopted in this paper is a modified one originally proposed by Kwakernaak(1978). Furthermore, we call the family of subsets defined by (3) as the set representation of a fuzzy set $\tilde{U}$. This view is slightly but not essentially different from its usual definition (see e.g. Kruse and Meyer(1987) or Kruse, Gebhardt and Klawonn(1994)).

As seen from the definition of fuzzy sets given by (1), there may be many fuzzy sets which have same strong cuts and level sets at every level $\alpha \in(0,1)$. This causes the following definition of the equivalence of fuzzy sets:

Definition 2.1 Two fuzzy sets $\tilde{U}$ and $\tilde{V}$ is called equivalent and denoted by $\tilde{U}=\tilde{V}$, if

$$
L_{\alpha} \tilde{U}=L_{\alpha} \tilde{V} \text { and } \quad L_{\bar{\alpha}} \tilde{U}=L_{\bar{\alpha}} \tilde{V} \quad \text { for } \forall \alpha \in(0,1) .
$$

Let $\left\{\alpha_{r}^{+} \mid r=1,2, \cdots\right\}$ and $\left\{\alpha_{r}^{-} \mid r=1,2, \cdots\right\}$ be a strictly increasing and decreasing sequence respectively such that

$$
\alpha=\lim _{r \rightarrow \infty} \alpha_{r}^{+} \text {and } \alpha=\lim _{r \rightarrow \infty} \alpha_{r}^{-}
$$

as well as $\alpha_{r}^{-} \in(\alpha, 1)$ and $\alpha_{r}^{+} \in(0, \alpha)$. Then, it can be shown that the following equalities hold:

$$
L_{\alpha} \tilde{U}=\bigcup_{r=1}^{\infty} \tilde{U}_{\alpha_{\bar{r}}} \text { for } \alpha \in[0,1)
$$

and

$$
L_{\bar{\alpha}} \tilde{U}=\bigcap_{r=1}^{\infty} \tilde{U}_{\alpha_{r}^{+}} \text {for } \alpha \in(0,1] .
$$

Let the basic space $\mathrm{X}$ be the Euclidean space $\mathrm{R}^{n}$, Then, the collection of all fuzzy sets in $\mathrm{R}^{n}$ is denoted by $\mathcal{G}\left(\mathrm{R}^{n}\right)$ when it satisfies that: (i) $\bigcap_{\alpha \in(0,1)} \tilde{u}_{\alpha} \neq \emptyset$; (ii) $\tilde{u}_{\alpha}$ is compact for each $\alpha \in(0,1)$; and (iii) supp. $\tilde{u}$ defined by supp. $\tilde{u}=$ cl. $\bigcup_{\alpha \in(0,1)} \tilde{u}_{\alpha}=$ cl. $L_{0} \tilde{u}$ is also compact. The family of fuzzy sets is denoted by $\mathcal{G}_{c}\left(\mathrm{R}^{n}\right)$ when each element $\tilde{u}$ in $\mathcal{G}_{c}\left(\mathrm{R}^{n}\right)$ is the member of $\mathcal{G}\left(\mathrm{R}^{n}\right)$ and its set representation is composed of compact and convex sets. The fuzzy sets in $\mathcal{G}_{c}\left(\mathrm{R}^{n}\right)$ is called ( $n$-dimensional) fuzzy vectors in this paper.

\section{Fuzzy Random Vectors}

Let $(\Omega, \mathcal{A}, P)$ be a complete probability space, where $\mathcal{A}$ is the $\sigma$-algebra generated by the subsets of $\Omega$, and $P$ is a nonatomic probability measure. Let $x_{0} \in \mathrm{R}^{n}$ be a random vector defined on this probability space. Assume now that we perceive the value of $x_{\mathrm{o}}$ through a set of rectangles $\left\{W_{i} \mid\right.$ $i \in J\}$ with $J$ a finite or countable set, each representing a rectangle in $\mathrm{R}^{n}$, such that $W_{i} \cap W_{j}=\emptyset$ for $i \neq j$ and $\bigcup_{i \in J} W_{i}=\mathrm{R}^{n}$. Let us define the special type of fuzzy set given by

$$
\tilde{I}_{i}=\left(\mathrm{R}^{n},\left\{\tilde{I}_{i, \alpha} \mid \alpha \in(0,1)\right\}, s_{\tilde{I}_{i}}\right),
$$

where $s_{\tilde{I}_{i}}(x)=\left\{x\right.$ coincides with $x_{\mathrm{o}}$ in $\left.W_{i}\right\}$ with $\tilde{I}_{i, \alpha}=W_{i}$ for $\forall \alpha \in(0,1)$, and

$$
t\left(s_{\tilde{I}_{i}}(x)\right)=\left(\tilde{I}_{i}\right)(x)= \begin{cases}1 & \text { if } x \in W_{i} \\ 0 & \text { otherwise. }\end{cases}
$$

Then, we can consider that in what rectangle $W_{i}$ the point $x_{\mathrm{o}}$ exists is equivalent to get the fuzzy set defined by (11). Therefore, the mapping $\tilde{x}: \Omega \rightarrow\left\{\tilde{I}_{i} \mid i \in J\right\}$ characterizes a special type of FRVCs, and this implies we can define FRVCs replacing the set representations $\left\{\tilde{I}_{i, \alpha} \mid \alpha \in(0,1)\right\}(i \in J)$ by the set of general set representations of fuzzy sets.

In order to define FRVCs mathematically, first define the reduced probability space $(\tilde{\Omega}, \tilde{\mathcal{A}}, \tilde{P})$ generated by $\mathcal{F} \subset \mathcal{A}$, where $\tilde{\Omega}$ is the quotient space of $\Omega$, i.e., $\tilde{\Omega}=\Omega / \sim$ with the equivalence relation, $\omega_{1} \sim \omega_{2} \Longleftrightarrow\left[\forall A \in \mathcal{F}, \omega_{1} \in\right.$ $\left.\mathrm{A} \Leftrightarrow \omega_{2} \in \mathrm{A}\right] ; \tilde{\mathcal{A}}$ is the reduction of $\mathcal{A}$ defined by $\tilde{\mathcal{A}}=$ $\left\{\tilde{\mathrm{A}} \mid c^{-1}(\tilde{\mathrm{A}}) \in \mathcal{A}\right\}$, where $c(\cdot)$ is the canonical projection, i.e., $c\left(\omega_{1}\right)=c\left(\omega_{2}\right)=\tilde{\omega} \in \tilde{\Omega}$ if $\underset{\tilde{A}}{\omega_{1}} \sim \omega_{2}\left(\omega_{1}, \omega_{2} \in \Omega\right)$; and $\tilde{P}$ is the probability measure on $\tilde{\mathcal{A}}$ given by $\tilde{P}(\tilde{\mathrm{A}})=P\left(c^{-1}(\tilde{\mathrm{A}})\right)$ for $\forall \tilde{\mathrm{A}} \in \tilde{\mathcal{A}}$. Let $\mathcal{K}_{c}^{n}$ be the collection of all the non-empty compact convex subsets of $\mathrm{R}^{n}$, and $\mathcal{B}\left(\mathcal{K}_{c}^{n}\right)$ stands for the Borel family of $\mathcal{K}_{c}^{n}$, i.e., the minimum $\sigma$-algebra generated by the open subsets of $\mathcal{K}_{c}^{n}$, where the topology of $\mathcal{K}_{c}^{n}$ is generated by the Hausdorff metric(see e.g. Klein and Thompson(1988)). Then, we have following definition:

Definition 3.1 The FRVC $\tilde{x}$ obtained as the vague perception of a ordinary random vector $x_{\circ}$ is defined by

$$
\tilde{x}: \tilde{\Omega} \rightarrow \mathcal{G}_{c}\left(\mathrm{R}^{n}\right),
$$

when its set representation $\left\{\tilde{x}_{\alpha}(\tilde{\omega}) \mid \tilde{\omega} \in \tilde{\Omega}, \alpha \in(0,1)\right\}$ is composed of $\tilde{\mathcal{A}}$-measurable compact convex random sets, i.e., $\tilde{\mathcal{A}}$-measurable compact-valued convex correspondences from $\tilde{\Omega}$ to $\mathrm{R}^{n}$. The measurability of $\tilde{x}$ is given through that of the function $\tilde{x}_{\alpha}$ from $\tilde{\Omega}$ to $\mathcal{B}\left(\mathcal{K}_{c}^{n}\right)$, i.e.,

$$
\tilde{x}_{\alpha}^{-1}(\mathrm{E}) \in \tilde{\mathcal{A}} \quad \text { for every } \mathrm{E} \in \mathcal{B}\left(\mathcal{K}_{c}^{n}\right) .
$$

The random vector $x_{0}$, of which the FRVC $\tilde{x}$ is a fuzzy perception, is called the original random vector of the FRVC. We denote the admissible set of original random vectors by $\chi$. Throughout this paper, we assume that the following conditions hold:

(A-1) $x_{\circ}$ (true original random vector) $\in$ supp. $\tilde{x}$ (A-2) $\left\{\xi \mid(\xi \in \chi) \wedge\left(\xi \in \bigcap_{\alpha \in(0,1)} \tilde{x}_{\alpha}\right.\right.$ a.s. in $\left.\left.\Omega\right)\right\} \neq \emptyset$ 


\section{Expectations of FRVCs}

Let $\xi$ be a possible original random variable of the FRVC $\tilde{x}$, i.e., a random vector in $\chi$. In order to investigate statistical properties of $\tilde{x}$, consider first the following statement:

$$
s_{\tilde{x}}(\xi)=\left\{\xi(\omega)=x_{\mathrm{o}}(\omega) \text { a.s. in } \Omega\right\} .
$$

Then, the above statement $s_{\tilde{x}}(\xi)$ may be rewritten by

$$
\begin{aligned}
& s_{\tilde{x}}(\xi)=\bigwedge\left\{\xi(\omega)=x_{\mathrm{o}}(\omega) \mid \omega \in \Omega\right. \text { except for } \\
& \left.\forall \omega \in \mathrm{A} \in \mathcal{A} \text { such that } \xi(\omega) \neq x_{\mathrm{o}}(\omega) \text { and } P(\mathrm{~A})=0\right\} .
\end{aligned}
$$

Using the results given by Bellman and Giertz(1973), it can be found that the truth value of $s_{\tilde{x}}(\xi)$ is evaluated as

$$
t\left(s_{\tilde{x}}(\xi)\right)=\underset{\omega \in \Omega}{\operatorname{essinf}}\{(\tilde{x}(\tilde{\omega}))(\xi(\omega))\},
$$

where

$$
\begin{aligned}
& \underset{\omega \in \Omega}{\operatorname{essinf}}\{(\tilde{x}(\tilde{\omega}))(\xi(\omega)\}= \\
& \quad \inf \{(\tilde{x}(\tilde{\omega}))(\xi(\omega)) \mid \omega \in \Omega, \tilde{\omega}=c(\omega) \text { except for } \\
& \left.\forall \omega \in A \in \mathcal{A} \text { such that } \xi(\omega) \neq x_{\circ}(\omega) \text { and } P(A)=0\right\} .
\end{aligned}
$$

By the way, the statement

$$
s_{E\{\tilde{x}\}}(x)=\left\{x \text { coincides with } E\left\{x_{\mathrm{o}}\right\}\right\}
$$

may be given by

$$
s_{E\{\tilde{x}\}}(x)=\bigvee_{\xi \in \chi}\left\{s_{\tilde{x}}(\xi) \bigwedge(E\{\xi\}=x)\right\} .
$$

Then, the truth value of the composite proposition $s_{E\{\tilde{x}\}}(x)$ is given by

$$
\begin{aligned}
& t\left(s_{E\{\tilde{x}\}}(x)\right)=t\left(\bigvee_{\xi \in \chi}\left\{s_{\tilde{x}}(\xi) \bigwedge(E\{\xi\}=x)\right\}\right) \\
& =\sup _{\xi \in \chi}\{(\underset{\omega \in \Omega}{\operatorname{esinf}}(\tilde{x}(\tilde{\omega}))(\xi(\omega))) \bigwedge(E\{\xi\}=x)\}
\end{aligned}
$$

with $\sup \emptyset=0$.

We introduce here the Aumann-like integral(abbreviated as AL-integral) given by

$$
\text { (AL) } \int \tilde{x}_{\alpha} d P=\left\{x \mid x=\int \xi d P, \quad \xi \in S\left(\tilde{x}_{\alpha}\right)\right\},
$$

where $\tilde{x}_{\alpha}$ is arbitrary element of the set representation of $\tilde{x}$, and $S\left(\tilde{x}_{\alpha}\right)$ is the selection set of $\tilde{x}_{\alpha}$, i.e.,

$S\left(\tilde{x}_{\alpha}\right)=\left\{\xi \mid \xi \in \chi ; \xi(\omega) \in \tilde{x}_{\alpha}(\tilde{\omega})\right.$ a.s. in $\left.\Omega ; \tilde{\omega}=c(\omega)\right\}$.

Using AL-integral given above, and defining the expectation of $\tilde{x}_{\alpha}$ by

$$
E\left\{\tilde{x}_{\alpha}\right\}=(\mathrm{AL}) \int \tilde{x}_{\alpha} d P,
$$

we can confirm the following property holds:

Proposition 4.1 Let $\tilde{x}$ be a FRVC and $\left\{\tilde{x}_{\alpha} \mid \alpha \in(0,1)\right\}$ be its set representation. Assume that every element of $\chi$ is integrable. Then,

$$
\left\{x \mid t\left(s_{E\{\tilde{x}\}}(x)\right)>\alpha\right\} \subseteq E\left\{\tilde{x}_{\alpha}\right\} \subseteq\left\{x \mid t\left(s_{E\{\tilde{x}\}}(x)\right) \geq \alpha\right\}
$$$$
\text { for } \forall \alpha \in(0,1) \text {. }
$$

Therefore, the following definition of the expectation of a FRVC $\tilde{x}$ may be reasonable:

Definition 4.1 Let $\tilde{x}$ be a FRVC and $\left\{\tilde{x}_{\alpha} \mid \alpha \in(0,1)\right\}$ be its set representation. Assume that every element of $\chi$ is integrable. Then, the expectation of the FRVC $\tilde{x}$ is defined by

$$
E\{\tilde{x}\}=\left(\mathrm{R}^{n},\left\{E\left\{\tilde{x}_{\alpha}\right\} \mid \alpha \in(0,1)\right\}, s_{E\{\tilde{x}\}}\right),
$$

where $s_{E\{\tilde{x}\}}$ is the proposition associated with the statement given by (18); and $\left\{E\left\{\tilde{x}_{\alpha}\right\} \mid \alpha \in(0,1)\right\}$ is the set representation of $E\{\tilde{x}\}$ given through (23).

It is clear from (24) that

$$
L_{\alpha} E\{\tilde{x}\} \subseteq E\left\{\tilde{x}_{\alpha}\right\} \subseteq L_{\bar{\alpha}} E\{\tilde{x}\} \quad \text { for } \forall \alpha \in(0,1),
$$

which means that $\left\{E\left\{\tilde{x}_{\alpha}\right\} \mid \alpha \in(0,1)\right\}$ satisfies indeed the condition imposed on set representations of fuzzy sets. The following corollary obtained immediately from the proof of Proposition 4.1

Corollary 4.1 Let $\tilde{x}$ be a FRVC and $\left\{\tilde{x}_{\alpha} \mid \alpha \in(0,1)\right\}$ be its set representation. Assume that every element of $\chi$ is integrable. Then $\left\{E\left\{L_{\alpha} \tilde{x}\right\} \mid \alpha \in(0,1)\right\}$ and $\left\{E\left\{L_{\bar{\alpha}} \tilde{x}\right\} \mid \alpha \in(0,1)\right\}$ are the set representations of the FRVCs equivalent to $E\{\tilde{x}\}$, and they satisfy

$$
L_{\alpha} E\{\tilde{x}\} \subseteq E\left\{L_{\alpha} \tilde{x}\right\} \subseteq E\left\{L_{\bar{\alpha}} \tilde{x}\right\} \subseteq L_{\bar{\alpha}} E\{\tilde{x}\} .
$$

Proposition 4.2 Let $\tilde{x}$ be a FRVC and the admissible set of possible original random variables $\chi$ be also convex, i.e., if $\xi_{1} \in \chi$ and $\xi_{2} \in \chi$, then $\xi=\lambda \xi_{1}+(1-\lambda) \xi_{2} \in \chi$ for $\lambda \in[0,1]$. Assume that $E\{\tilde{x}\}$ exists. Then, $E\{\tilde{x}\}$ is convex.

Theorem 4.1 Let $\tilde{x}$ be a fuzzy vector, i.e., $\tilde{x} \in \mathcal{G}_{c}\left(\mathrm{R}^{n}\right)$, and assume that the admissible class of possible original random vectors $\chi$ satisfies (i) $\chi$ is convex; (ii) $\chi$ is composed of the random vectors satisfying $\|\xi(\omega)\| \leq h(\omega)$ a.s. in $\Omega$, where $h$ 
is a random variable such that $\int h d P<+\infty$; and (iii) $\chi$ is closed with respect to convergent sequences, i.e., if $u_{j} \rightarrow u$ a.s. in $\Omega\left(u_{j} \in \chi\right)$, then $u$ is the element of $\chi$. Then, $E\{\tilde{x}\}$ is a fuzzy vector, i.e. $E\{\tilde{x}\} \in \mathcal{G}_{c}\left(\mathrm{R}^{n}\right)$.

In order to investigate further the properties of the expected FRVCs, we have to introduce the concept of integrably boundedness of FRVCs.

Definition 4.2 The admissible set of possible original random vectors $\chi$ is $L^{n}$-integrably bounded if $\chi$ consists of all the random vectors satisfying $\|\xi(\omega)\|^{n} \leq h(\omega)$ a.s. in $\Omega$, where $h(\omega)$ is the random variable such that $\int h d P<+\infty$.

Theorem 4.2 Let $\tilde{x}$ be a fuzzy vector. Assume that $\chi$ is $L^{1}$ integrably bounded. Then, $E\{\tilde{x}\}$ is a fuzzy vector, and it satisfies

$$
\text { cl. } L_{\alpha} E\{\tilde{x}\}=E\left\{\operatorname{cl} . L_{\alpha} \tilde{x}\right\} \quad \text { for } \forall \alpha \in[0,1)
$$

and

$$
L_{\bar{\alpha}} E\{\tilde{x}\}=E\left\{L_{\bar{\alpha}} \tilde{x}\right\} \quad \text { for } \forall \alpha \in(0,1] .
$$

\section{Conclusions}

In this paper, a class of FRVCs and their expectations have been introduced from the consistent viewpoint of fuzzy perceptions of ordinary non-vague random vectors, and some properties of FRVCs have been examined theoretically, where set representations of fuzzy sets are thoroughly adopted because of the feasibility for describing operations between FRVCs.

It may be fair to say that by adopting the family of compact convex correspondences as the set representation of a FRVC, we have been able to introduce its reasonable definition, which is basically compatible with (scalar) fuzzy random variables proposed by Kwakernaak(1978) and is never obtained from their simple extension. Furthermore, the proposed concept of FRVCs as vague perceptions of random vectors may be, in some sense, a bridge between two major types of fuzzy random variables, one is proposed by Kwakernaak and another is by Puri and Ralescu(1986).

\section{References}

R.J. Aumann(1965) Integrals of set-valued functions, Journal of Mathematical Analysis and its Applications, 12, 1-12.

R.E. Bellman and M. Giertz(1973) On the analytic formalism of the theory of fuzzy sets, Information Sciences, 5, 149-156.

S.B. Boswell and M.S. Taylor(1987) A central limit theorem for fuzzy random variables, Fuzzy Sets and Systems, 24, 331-344.

T. Fukuda(1994) On a class of vaguely dependent random variables, In First Asian Control Conference, 1, 475-478

T. Fukuda(1995a) On a class of fuzzy random vectors obtained as vague perception of random phenom- ena, In International Joint Conference of 4 th IEEE Int. Conf. on Fuzzy Systems and Int. Fuzzy Eng. Symp.(FUZZ/IEEE/IFES'95), II, 963-970.

T. Fukuda(1995b) Some properties of a class of fuzzy random vectors, In The 26th ISCIE International Symposium on Stochastic Systems Theory and its Applications, pages 131-136.

H. Inoue(1991) A strong law of large numbers for fuzzy random sets, Fuzzy Sets and Systems, 41, 285-291.

E.Klein and A.C. Thompson(1988) Theory of Correspondences, John Wiley \& Sons, Netherlands.

E.P. Klement, M.L. Puri, and D.A. Ralescu(1986) Limit theorems for fuzzy random variables, Proceedings of Royal Society of London, A407, 171-182.

R. Kruse and K. D. Meyer(1987) Statistics with Vague Data, D. Reidel Publishing Company, Dordecht.

R. Kruse, J. Gebhardt and F. Klawonn(1994) Foundations of Fuzzy Systems, John Wiley \& Sons, Chichester.

H. Kwakernaak(1978) Fuzzy random variables-I: definitions and theorems, Information Sciences, 15, 1-29.

G. Matheron(1975) Random Sets and Integral Geometry, John-Wiley, New York.

M. Miyakoshi and M. Simbo(1984) A strong law of large numbers for fuzzy random variables, Fuzzy Sets and Systems, 12, 133-142.

M.L. Puri and D.A. Ralescu(1986) Fuzzy random variables, Journal of Mathematical Analysis and its Applications, 114, 409-422.

T. Uemura(1993) A law of large numbers for random sets, Fuzzy Sets and Systems, 59, 181-188.

\section{A. Proofs of Propositions and Theorems}

\section{Proof of Proposition 4.1}

It is clear from (3),(10) and the definition of the selection set given in (22) that

$$
L_{\overline{1}} \tilde{x} \subseteq L_{\alpha} \tilde{x} \subseteq \tilde{x}_{\alpha} \subseteq L_{\bar{\alpha}} \tilde{x} \text { for each } \alpha \in(0,1) .
$$

Then, under the assumption (A-2) we have that

$$
\emptyset \neq S\left(L_{\alpha} \tilde{x}\right) \subseteq S\left(\tilde{x}_{\alpha}\right) \subseteq S\left(L_{\bar{\alpha}} \tilde{x}\right),
$$

which follows

$$
(\mathrm{AL}) \int L_{\alpha} \tilde{x} d P \subseteq(\mathrm{AL}) \int \tilde{x}_{\alpha} d P \subseteq(\mathrm{AL}) \int L_{\bar{\alpha}} \tilde{x} d P,
$$

where the existence of each integral is guaranteed because that the admissible class of possible originals $\chi$ is assumed to be composed of integrable random vectors.

(i) If there exists $\zeta$ such that

$$
\zeta \in\left\{x \mid t\left(s_{E\{\tilde{x}\}}(x)\right)>\alpha\right\}
$$

there is some element $\xi$ of $\chi$ satisfying $E\{\xi\}=\zeta$ and

$$
\xi(\omega) \in L_{\alpha} \tilde{x}(\tilde{\omega}) \quad \text { a.s. in } \Omega .
$$


It is clear that $\xi$ in $(34)$ is the element of $S\left(L_{\alpha} \tilde{x}\right)$ and hence we have

$$
\zeta=E\{\xi\}=\int \xi d P \in(\mathrm{AL}) \int L_{\alpha} \tilde{x} d P
$$

or equivalently we can conclude that

$$
\left\{x \mid t\left(s_{E\{\tilde{x}\}}(x)\right)>\alpha\right\} \subseteq(\mathrm{AL}) \int L_{\alpha} \tilde{x} d P=E\left\{L_{\alpha} \tilde{x}\right\} .
$$

(ii) If there exists $\zeta$ such that

$$
\zeta \in(\mathrm{AL}) \int L_{\bar{\alpha}} \tilde{x} d P=\left\{x \mid x=\int \xi d P, \xi \in S\left(L_{\bar{\alpha}} \tilde{x}\right)\right\},
$$

there is some element $\xi$ of $\chi$ satisfying $\zeta=\int \xi d P$ and

$$
(\tilde{x}(\tilde{\omega}))(\xi(\omega)) \geq \alpha \quad \text { a.s. in } \Omega .
$$

(38) implies that there exists $\xi \in \chi$ satisfying $\zeta=E\{\xi\}$ and

$$
\underset{\omega \in \Omega}{\operatorname{essinf}}(\tilde{x}(\tilde{\omega}))(\xi(\omega)) \geq \alpha,
$$

which means that

$$
\zeta \in\left\{x \mid t\left(s_{E\{\tilde{x}\}}(x)\right) \geq \alpha\right\},
$$

or equivalently

$$
(\mathrm{AL}) \int L_{\bar{\alpha}} \tilde{x} d P=E\left\{L_{\bar{\alpha}} \tilde{x}\right\} \subseteq\left\{x \mid t\left(s_{E\{\tilde{x}\}}(x)\right) \geq \alpha\right\} .
$$

(iii) From (32), (36) and (41), we have

$$
\begin{aligned}
\left\{x \mid t\left(s_{E\{\tilde{x}\}}(x)\right)>\alpha\right\} \subseteq E\left\{L_{\alpha} \tilde{x}\right\} \subseteq E\left\{\tilde{x}_{\alpha}\right\} \\
\subseteq E\left\{L_{\bar{\alpha}} \tilde{x}\right\} \subseteq\left\{x \mid t\left(s_{E\{\tilde{x}\}}(x)\right) \geq \alpha\right\}
\end{aligned}
$$

for each $\alpha \in(0,1)$.

\section{Proof of Proposition 4.2}

Let $x_{1}$ and $x_{2}$ be the elements of $E\left\{\tilde{x}_{\alpha}\right\}$. Then, one can find the two random vectors $\xi_{1}$ and $\xi_{2}$ in $S\left(\tilde{x}_{\alpha}\right)$ satisfying $x_{1}=E\left\{\xi_{1}\right\}$ and $x_{2}=E\left\{\xi_{2}\right\}$. Define here the random vector $\xi_{\lambda}$ by

$$
\xi_{\lambda}(\omega)=\lambda \xi_{1}(\omega)+(1-\lambda) \xi_{2}(\omega) \text { for every } \lambda \in[0,1] .
$$

Then, it is clear $\xi_{\lambda}(\omega) \in \chi$ by the convexity of $\chi$ and $\xi_{\lambda}(\omega) \in$ $\tilde{x}_{\alpha}(\omega)$ a.s. in $\Omega$ from the convexity of $\tilde{x}_{\alpha}$, which means $\xi_{\lambda} \in$ $S\left(\tilde{x}_{\alpha}\right)$ and $x_{\lambda}$ defined by $x_{\lambda}=E\left\{\xi_{\lambda}\right\}$ is the element of $E\left\{\tilde{x}_{\alpha}\right\}$. Furthermore, using (43), it follows

$$
x_{\lambda}=\lambda x_{1}+(1-\lambda) x_{2} \in E\left\{\tilde{x}_{\alpha}\right\},
$$

which means that $E\left\{\tilde{x}_{\alpha}\right\}$ is convex.

\section{Proof of Theorem 4.1}

In order to prove Theorem 4.1, the following lemmas are needed(Klein and Thompson,1988):

Lemma A.1 Let $\left\{\mathrm{A}_{r} \mid r=1,2, \cdots\right\}$ be the constant sequence of subsets of $\mathrm{R}^{n}$, i.e., $\mathrm{A}_{r}=\mathrm{A}_{\mathrm{o}}$ for all $r=1,2, \cdots$. Then, $\lim _{r \rightarrow \infty} \mathrm{A}_{r}$. exists and $\lim _{r \rightarrow \infty} \mathrm{A}_{r}=\mathrm{cl}$. $\mathrm{A}_{\mathrm{o}}$.

Lemma A.2 Let $\left\{f_{r} \mid r=1,2, \cdots\right\}$ be a sequence of $(\mathcal{A}$ measurable ) integrable functions such that

$$
\left\|f_{r}(\omega)\right\| \leq h(\omega) \quad \text { a.s. in } \Omega \text { for } r=1,2, \cdots
$$

and $\int f_{r} d P \rightarrow \xi$. Then, there is an integrable function $f$ satisfying

$$
\xi=\int f d P=\lim _{r \rightarrow \infty} \int f_{r} d P
$$

with $f(\omega) \in \limsup _{r \rightarrow \infty}\left\{f_{r}(\omega)\right\}$ a.s. in $\Omega$.

Note that lim, liminf and limsup in Lemmas A.1 and A.2 are given in the sense of Kuratowski.

\section{(Proof of Theorem 4.1)}

Throughout the proof, let $\left\{\tilde{x}_{\alpha} \mid \alpha \in(0,1)\right\}$ be the set representation of $\tilde{x}$.

(i) Let $\left\{u_{r}(\tilde{\omega}) \mid r=1,2, \cdots: \tilde{\omega} \in \tilde{\Omega}\right\}$ be a sequence of correspondences from $\tilde{\Omega}$ to $\mathrm{R}^{n}$ with the property that there is some integrable function $h(\omega)$ such that $\|x(\omega)\| \leq h(\omega)$ a.s. in $\Omega$ for each $x \in S\left(u_{r}\right)$, where

$$
S\left(u_{r}\right)=\left\{\eta \mid \eta \in \chi, \eta(\omega) \in u_{r}(\tilde{\omega}) \text { a.s. in } \Omega\right\} .
$$

Assume that

$$
\zeta \in \limsup _{r \rightarrow \infty}\left\{\zeta_{r}\right\}, \quad \text { where } \zeta_{r}=\left\{(\mathrm{AL}) \int u_{r} d P\right\} .
$$

Then, there is a subsequence $\left\{\zeta_{r_{j}} \mid j=1,2, \cdots\right\}$ with

$$
\zeta=\lim _{j \rightarrow \infty} \zeta_{r_{j}}=\lim _{j \rightarrow \infty} \int \varphi_{j} d P \quad\left(\varphi_{j} \in S\left(u_{r_{j}}\right)\right)
$$

because that $\zeta$ is a cluster point of $\left\{\zeta_{r} \mid r=1,2, \cdots\right\}$. Applying Lemma A.2 to $\left\{\varphi_{j}\right\}$ given by (49), we can confirm that there is an integrable function $\varphi$ with

$$
\zeta=\int \varphi d P=\lim _{j \rightarrow \infty} \int \varphi_{j} d P
$$

and

$$
\varphi \in \limsup _{j \rightarrow \infty}\left\{\varphi_{j}\right\} \subseteq S\left(\limsup _{r \rightarrow \infty} u_{r}\right) \quad \text { a.s. in } \Omega
$$


because of the condition (iii). Hence, we have

$$
\limsup _{r \rightarrow \infty}\left\{(\mathrm{AL}) \int u_{r} d P\right\} \subseteq(\mathrm{AL}) \int \limsup _{r \rightarrow \infty} u_{r} d P .
$$

(ii) For $r=1,2, \cdots$, set

$$
\tilde{x}_{\alpha, r}(\tilde{\omega})=\tilde{x}_{\alpha}(\tilde{\omega}) \cdot \quad \text { a.s. in } \tilde{\Omega} .
$$

Since $\tilde{x}_{\alpha}(\tilde{\omega})$ is compact and all elements of $\chi$ are integrable, it follows from Lemma A.1 that

$$
\lim _{r \rightarrow \infty} \tilde{x}_{\alpha, r}(\tilde{\omega})=\operatorname{cl} . \tilde{x}_{\alpha}(\tilde{\omega})=\tilde{x}_{\alpha}(\tilde{\omega}) \quad \text { a.s. in } \tilde{\Omega}
$$

and

$$
\lim _{r \rightarrow \infty} E\left\{\tilde{x}_{\alpha, r}\right\}=\operatorname{cl} . E\left\{\tilde{x}_{\alpha}\right\} .
$$

Then, using (52), it can be found that

$$
\begin{aligned}
\operatorname{cl} . E\left\{\tilde{x}_{\alpha}\right\} & =\limsup _{r \rightarrow \infty}\left\{(\mathrm{AL}) \int \tilde{x}_{\alpha, r} d P\right\} \\
& \subseteq(\mathrm{AL}) \int \limsup _{r \rightarrow \infty} \tilde{x}_{\alpha, r} d P \\
& =(\mathrm{AL}) \int \operatorname{cl} . \tilde{x}_{\alpha} d P \\
& =E\left\{\tilde{x}_{\alpha}\right\},
\end{aligned}
$$

which means that $E\left\{\tilde{x}_{\alpha}\right\}$ is closed. On the other hand, since each element of $\chi$ is bounded by the integrable real-valued function because of the condition (ii), it follows that each element of $E\left\{\tilde{x}_{\alpha}\right\}$ is also bounded. Therefore, $E\left\{\tilde{x}_{\alpha}\right\}$ is compact.

(iii) It is clear from (A-2) that $E\left\{\tilde{x}_{\alpha}\right\} \neq \emptyset$ for each $\alpha \in(0,1)$ which yields $\bigcap_{\alpha \in(0,1)} E\left\{\tilde{x}_{\alpha}\right\} \neq \emptyset$, and using same procedure as that given in (ii), we can confirm supp. $\tilde{x}$ is also compact. Furthermore, from Proposition 4.2, we know that $E\left\{\tilde{x}_{\alpha}\right\}$ is convex with the condition (i). Therefore, combining the results of the step (ii) with this step (iii), we can conclude that $E\{\tilde{x}\}$ satisfies all the conditions for $\mathcal{G}_{c}\left(\mathrm{R}^{n}\right)$.

\section{Proof of Theorem 4.2}

In order to prove Theorem 4.2, we need the following lemma(Klein and Thompson, 1988):

Lemma A.3 Let $\left\{u_{r} \mid r=1,2, \cdots\right\}$ be a sequence of $\tilde{\mathcal{A}}$ measurabel $L^{1}$-integrably bounded correspondences. Then

$$
(\mathrm{AL}) \int \liminf u_{r} d P \subseteq \liminf \left\{(\mathrm{AL}) \int u_{r} d P\right\} .
$$

It should be noted that with condition that $\chi$ is $L^{1}$-integrable bounded, the AL-integral of $u_{r}$ coincides completely with its Aumann integral proposed by Aumann(1965)
When $\chi$ is $L^{1}$-integrably bounded, it is clear that the conditions (i), (ii) and (iii) in Theorem 4.1 are satisfied. Hence, the remainder is to prove (28) and (29).

From (9), we have

$$
L_{\alpha} \tilde{x}(\tilde{\omega})=\bigcup_{r=1}^{\infty} \tilde{x}_{\alpha_{r}^{-}}(\tilde{\omega}) \quad \text { a.s. in } \tilde{\Omega}
$$

and

$$
L_{\alpha} E\{\tilde{x}\}=\bigcup_{r=1}^{\infty} E\left\{\tilde{x}_{\alpha_{r}^{-}}\right\}
$$

where $\left\{\alpha_{r^{-}}^{-}\right\}$is the sequence defined in (8). Since $\left\{\tilde{x}_{\alpha_{r}^{-}} \mid r=\right.$ $1,2, \cdots\}$ is the increasing sequence of correspondences, it can be found(see e.g. Matheron, 1975) that

$$
\begin{aligned}
& \lim _{r \rightarrow \infty} \tilde{x}_{\alpha_{r}^{-}}=\operatorname{cl} .\left(\bigcup_{r=1}^{\infty} \tilde{x}_{\alpha_{r}^{-}}(\tilde{\omega})\right)=\operatorname{cl} . L_{\alpha} \tilde{x}(\tilde{\omega}) \text { a.s. in } \tilde{\Omega} . \\
& \lim _{r \rightarrow \infty} E\left\{\tilde{x}_{\alpha_{r}^{-}}\right\}=\operatorname{cl} .\left(\bigcup_{r=1}^{\infty} E\left\{\tilde{x}_{\alpha_{r}^{-}}\right\}\right)=\operatorname{cl} . L_{\alpha} E\{\tilde{x}\} .
\end{aligned}
$$

Then, applying Lemma A.3 and (52) to (60) and (61), it follows that

$$
\begin{aligned}
& E\left\{\text { cl. } L_{\alpha} \tilde{x}\right\}=E\left\{\liminf _{r \rightarrow \infty} \tilde{x}_{\alpha_{r}^{-}}\right\} \subseteq \operatorname{cl} . L_{\alpha} E\{\tilde{x}\} \\
& =\limsup _{r \rightarrow \infty} E\left\{\tilde{x}_{\alpha_{r}^{-}}\right\} \subseteq E\left\{\limsup _{r \rightarrow \infty} \tilde{x}_{\alpha_{r}^{-}}\right\}=E\left\{\text { cl. } L_{\alpha} \tilde{x}\right\}
\end{aligned}
$$

which means (28) holds for every $\alpha \in(0,1)$.

It can be shown from (10) that

$$
L_{\bar{\alpha}} \tilde{x}(\tilde{\omega})=\bigcap_{r=1}^{\infty} \tilde{x}_{\alpha_{r}^{+}}(\tilde{\omega}) \text { for } \forall \tilde{\omega} \in \tilde{\Omega}
$$

and

$$
L_{\bar{\alpha}} E\{\tilde{x}\}=\bigcap_{r=1}^{\infty} E\left\{\tilde{x}_{\alpha_{r}^{+}}\right\}
$$

where $\left\{\alpha_{r}^{+}\right\}$is the sequence defined in (8). Since $\left\{\tilde{x}_{\alpha_{r}^{+}} \mid r=\right.$ $1,2, \cdots\}$ is the decreasing sequence of correspondences, it can be found(see e.g. Matheron, 1975) that

$$
\lim _{r \rightarrow \infty} \tilde{x}_{\alpha_{r}^{+}}=\left(\bigcap_{r=1}^{\infty} \tilde{x}_{\alpha_{r}^{+}}(\tilde{\omega})\right)=L_{\bar{\alpha}} \tilde{x}(\tilde{\omega}) \text { a.s. in } \tilde{\Omega} .
$$

and

$$
\lim _{r \rightarrow \infty} E\left\{\tilde{x}_{\alpha_{r}^{+}}\right\}=\left(\bigcap_{r=1}^{\infty} E\left\{\tilde{x}_{\alpha_{r}^{+}}\right\}\right)=L_{\bar{\alpha}} E\{\tilde{x}\} .
$$

Then, applying Lemma A.3 and (52), we can confirm (29) holds for every $\alpha \in(0,1)$.

(Proof of Theorem 4.2) 